\title{
Analysis of 16 different matrix metalloproteinases (MMP-1 to MMP-20) in the synovial membrane: different profiles in trauma and rheumatoid arthritis
}

\author{
Yrjö T Konttinen, Mia Ainola, Heikki Valleala, Jian Ma, Hideo Ida, Jami Mandelin, \\ Raimund W Kinne, Seppo Santavirta, Timo Sorsa, Carlos López-Otín, Michiaki Takagi
}

Department of Oral Medicine, University of Helsinki, Finland Y T Konttinen

\section{Department of}

Medicine, Helsinki

University Central

Hospital and

Department of

Anatomy, University of Helsinki, Finland

Y T Konttinen

M Jumppanen

$\mathrm{H}$ Valleala

$\mathrm{J}$ Ma

J Mandelin

Department of Orthopaedic Surgery, Yamagata University School of Medicine, Yamagata, Japan

$\mathrm{H}$ Ida

M Takagi

Experimental

Rheumatology,

Friedrich-Schiller-

University, Jena,

Germany

R W Kinne

Department of

Orthopaedics and

Traumatology,

Helsinki University

Central Hospital,

Helsinki, Finland

S Santavirta

Department of

Periodontology,

University of Helsinki,

Finland

T Sorsa

University of Oviedo, Oviedo, Spain

C López-Otin

Correspondence to: Dr Y T Konttinen, Institute of Biomedicine, Department of Anatomy, PO Box 9

(Siltavuorenpenger $20 \mathrm{~A}$ ),

FIN-00014 University of

Helsinki, Finland.

Accepted for publication

19 June 1999
Abstract

Objective-To define the pattern of mRNA expression of all human matrix metalloproteinases (MMPs) described to date in rheumatoid arthritis (RA) and traumatic synovial membrane, in order to differentiate between a physiological tissue remodelling pattern and that associated with inflammatory tissue destruction. Methods-Analysis of SwissProt protein and EMBL/GenBank nucleotide sequence banks, protein sequence alignment, reverse transcriptase-polymerase chain reaction and nucleotide sequencing were used.

Results-MMP-2 (gelatinase A), MMP-3 (stromelysin-1), MMP-11 (stromelysin-3) and MMP-19 were constitutively expressed. MMP-1 (fibroblast type collagenase), MMP-9 (gelatinase B) and MMP-14 (MT1-MMP) were expressed in all RA, but only in $55-80 \%$ of trauma samples. MMP-13 (collagenase-3) and MMP-15 (MT2-MMP) were expressed exclusively in RA (80-90\% of the samples). MMP-20 (enamelysin) was absent and MMP-8 (collagenase-2) was rarely found in RA or trauma. All other MMPs $(-7,-10$, $-12,-16,-17)$ had an intermediate pattern of expression.

Conclusions-Some MMPs without interstitial collagenase activity seem to have a constitutive pattern of expression and probably participate in physiological synovial tissue remodelling. Some MMPs are exclusively associated to RA synovitis, for example, MMP-13, which preferentially degrades type II collagen and aggrecan, and MMP-15, which activates proMMP-2 and proMMP-13 and is involved in tumour necrosis factor $\alpha$ processing. This clear cut rheumatoid/inflammatory MMP profile, more complex than has been previously appreciated, may facilitate inflammatory tissue destruction in RA.

(Ann Rheum Dis 1999;58:691-697)

Matrix metalloproteinases (MMPs) form a family of enzymes, which share a structurally similar domain structure, in particular the zinc dependent catalytic domain and the activation peptide (propeptide) thought to be responsible for the latency of the proMMP enzyme species. ${ }^{1}$ More specifically, the main domains of the MMPs are (1) the signal peptide responsible for the endosomal translocation of the enzyme protein; (2) the activation peptide or propeptide containing a cysteine at the position 73 , which is supposed to occupy the critical fourth coordination site of the active site zinc and is thus responsible for the latency of the proMMP species; (3) the catalytic domain, which contains the three conserved amino acids/ sequences binding the active site zinc; (4) the proline rich hinge region; and (5) the hemopexin-like domain (not found in MMP-7 (=matrilysin, PUMP-1)), which is supposed to play a part for the substrate specificity. In addition, gelatinases contain gelatin binding, fibronectin-like inserts in the catalytic domain and gelatinase B (MMP-9) contains a type V collagen-like domain. Finally, membrane type MMPs (MT-MMP) contain a C-terminal insertion with a transmembrane domain integrating them to the cell membrane, plus a domain between the propeptide and the catalytic domain. This domain is also expressed by MMP-11 (stromelysin-3) and is recognised by furin, a Golgi associated intracellular proMMP activator.

Because of the ability of the activated MMP enzymes to mount a nucleophilic/peptidolytic attack in the neutral $\mathrm{pH}$ prevailing in the extracellular space, and because of their substrate specificity, they are believed to play an important part in inflammatory, tissue destructive diseases, including rheumatoid arthritis (RA). Since the detection of the first member of the MMP species-that is, tadpole collagenase - they have attracted the interest of rheumatologists. Fibroblast type collagenase, later renamed $M M P-1$, was the first MMP detected in the rheumatoid synovial membrane. ${ }^{2}$ Later, also "neutrophil-type collagenase" or MMP-8 ${ }^{34}$ and stromelysin-1 or MMP-3, which was also called "proteoglycanase", ${ }^{5}$ have raised interest.

Most of the studies so far performed in rheumatoid arthritis (RA) have analysed only one member of the MMP family. However, a comparative analysis of the human MMP family tree, based on amino acid sequence data obtained from the protein and nucleotide sequence data banks, disclosed a tree with 16 branches (see fig 1). MMP-4, MMP-5 and MMP-6 have been only tentatively described, but not cloned and sequenced. According to currently registered sequence data, MMP-18 and MMP-19 seem to be identical, but are not. 


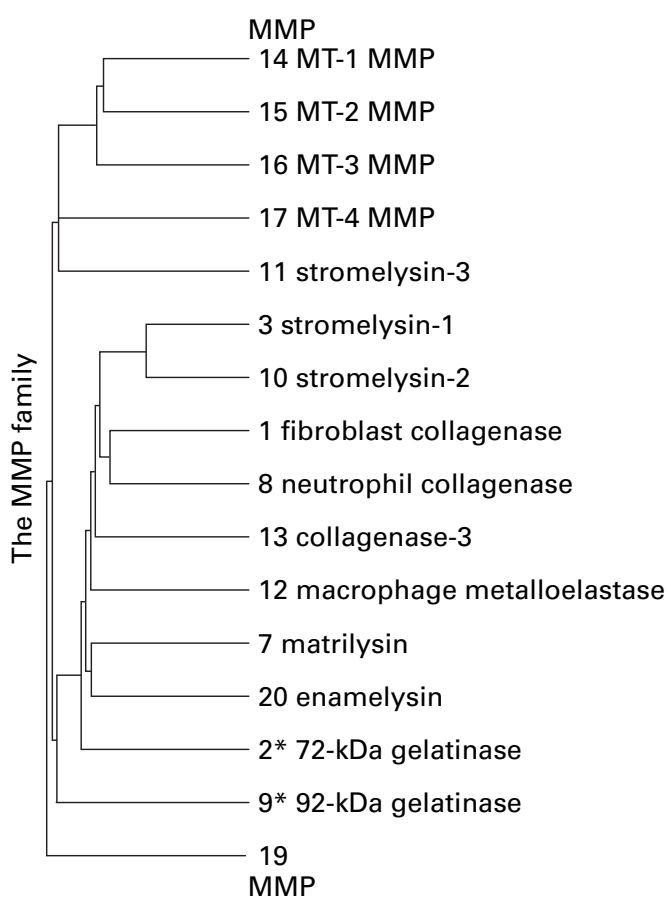

Figure 1 Family tree of the matrix metalloproteinases. Distance along the vertical axis in the family tree is proportional to the difference between sequences. The horizontal distance has no significance.

MMP-18 corresponds to Xenopus collagenase-4, an enzyme whose human homologue has not been identified. ${ }^{7}$ Without knowing this, Cossins and coworkers identified simultaneously "another" MMP-18 and described it as a putative novel human matrix metalloproteinase. ${ }^{8}$ This has been later characterised in more detail and is now known as MMP-19. ${ }^{9}$ These 16 human MMPs are often divided into subfamilies, usually termed interstitial collagenases, gelatinases, stromelysins, MT-MMPs, and others, although it is unclear whether this subdivision reflects the true degree of complexity.

Because of the rapid expansion of the human MMP family, well characterised and monospecific antibodies are scarcely available. Because of the obvious interest in comparing, not just individual MMPs, but the overall MMP profile in RA versus that of "non-inflammatory" conditions, we designed specific primers for each and every human MMP, to compare their expression in rheumatoid and traumatic synovial membrane samples. This study reports also on the primers that have been tested and found suitable for polymerase chain reaction studies on human MMPs.

\section{Methods}

FAMILY TREE OF THE MMPS AVAILABLE IN THE SWISSPROT PROTEIN SEQUENCE DATA BANK OR IN THE EMBL/GENBANK NUCLEOTIDE SEQUENCE BANK Protein sequences were aligned in a server Cypress by a metacomputer (Silicon Graphic Power Challenge) in the Center for Scientific Calculation (CSC), Finland. The GCG's (Genetics Computer Group, Madison, WI, USA) program used for the aligning (PileUp) creates a multiple sequence alignment using a simplification of the progressive alignment method of Feng and Doolittle. ${ }^{10}$ The method used is similar to that of Higgins and Sharp. ${ }^{11}$ The alignment procedure begins with the pairwise alignment of the two most similar sequences, producing a cluster of two aligned sequences. This cluster can be aligned to the next most related sequence or a cluster of aligned sequences. Two clusters of sequences can be aligned by a simple extension of the pairwise alignment of two individual sequences. The final alignment is achieved by a series of progressive, pairwise alignments that include increasingly dissimilar sequences and clusters, until all sequences have been included in the final pairwise alignment. Distance along the vertical axis in the family tree is proportional to the difference between the sequences (fig 1). The horizontal distance has no significance (Program Manual for the Wisconsin Package, Version 9, Genetics Computer Group, Madison, Wisconsin, USA). The matrix used in this alignment was blosum 62 , the gap creation penalty was set to 12 and the gap extension penalty to 4. Both gelatinases (MMP-2 and MMP-9) were modified so that the amino acid sequence between Val 221 and Asp 392 in MMP-2, and the sequence between Val 218 and Asp 390 in MMP-9 was removed. This modification was performed because the long gelatin binding sequence distorted the otherwise good aligning within the given penalty values. When less stringent values (10 and 2) were used, the aligning was not acceptable.

PATIENTS AND SAMPLES

The study design was approved by the Institutional Review Board of the Helsinki University Central Hospital. Recommendations of the Helsinki Declaration (Hong Kong amendment, September 1989) were followed. Synovial tissue specimens were obtained at synovectomy or diagnostic/therapeutic arthroscopy (see also table 1). Of the RA patients, ${ }^{12}$ eight were women and two men, with a mean age of 62 years (range 33 to 81). Samples were obtained from four knees, three hips, two elbows and one shoulder joint. Seven RA patients were taking non-steroidal, antiinflammatory drug medication, two were taking analgesic drug medication, and one patient did not use any non-steroidal, antiinflammatory drugs. Six RA patients were receiving a low dose $(10 \mathrm{mg}$ prednisolone or equivalent) corticosteroids. As to the disease modifying, anti-rheumatic drugs, six were receiving a single drug therapy (one methotrexate, two sulfasalazine, one D-penicillamine, one chloroquine, one azathioprine). Two patients had a combination treatment, one with sulfasalazine and methotrexate and one with cyclosporine, methotrexate and hydroxychloroquine. Two patients did not receive any disease modifying, anti-rheumatic drugs, but only low dose corticosteroids and nonsteroidal, anti-inflammatory drugs. Nine patients (seven women and two men, mean age 35 years, range 21 to 55 ) had traumatic or painful "non-inflammatory" knee disorders (that is, rupture of the meniscus $(n=2)$, chondromalacia patellae $(n=2)$, subluxation of the patella $(n=2)$, rupture of the anterior cruciate ligament $(n=1)$. The two remaining 
Table 1 Demographic and clinical data of patients, from whom synovial membrane samples were available for RT-PCR analysis of the matrix metalloproteinases

\begin{tabular}{|c|c|c|c|c|c|}
\hline Patient & Diagnosis & Sex & Age & foint operated & Medication \\
\hline 1 & RA & $\mathrm{F}$ & 68 & elbow & low dose peroral prednisolone, methotrexate \\
\hline 2 & RA & $\mathrm{F}$ & 81 & knee & tramadol, sulfasalazine \\
\hline 3 & RA & $\mathrm{F}$ & 61 & knee & NSAID, D-penicillamine \\
\hline 4 & RA & $\mathrm{F}$ & 77 & knee & NSAID, low dose peroral prednisolone, alendronate \\
\hline 5 & RA & $\mathrm{F}$ & 62 & knee & NSAID, low dose peroral prednisolone, sulfasalazine, methotrexate \\
\hline 6 & RA & $\mathrm{F}$ & 33 & hip & NSAID, hydroxychloroquine, methotrexate, cyclosporine A \\
\hline 7 & RA & $\mathrm{F}$ & 77 & hip & NSAID, chloroquine \\
\hline 8 & RA & $\mathrm{F}$ & 69 & elbow & NSAID, low dose peroral prednisolone, sulfasalazine \\
\hline 9 & RA & M & 49 & shoulder & NSAID, low dose peroral prednisolone \\
\hline 10 & RA & M & 44 & hip & tramadol, low dose peroral prednisolone, azathioprine \\
\hline 11 & rupture of the meniscus & $\mathrm{F}$ & 55 & knee & NSAID \\
\hline 12 & subluxation of the patella & $\mathrm{F}$ & 21 & knee & - \\
\hline 13 & chondromalacia patellae & $\mathrm{F}$ & 42 & knee & - \\
\hline 14 & chondromalacia patellae & $\mathrm{F}$ & 30 & knee & NSAID \\
\hline 15 & rupture of the ACL & $\mathrm{F}$ & 33 & knee & NSAID \\
\hline 16 & distensio genus & $\mathrm{F}$ & 37 & knee & - \\
\hline 17 & distensio genus & M & 29 & knee & - \\
\hline 18 & rupture of the meniscus & $\mathrm{F}$ & 27 & knee & NSAID \\
\hline 19 & subluxation of the patella & M & 41 & knee & - \\
\hline
\end{tabular}

$\mathrm{RA}=$ rheumatoid arthritis, $\mathrm{ACL}=$ anterior cruciate ligament $($ of the knee $), \mathrm{NSAID}=$ non-steroidal anti-inflammatory drug.

controls had knee injury and pain, but no arthroscopic and bioptic signs of specific disease). All samples were snap frozen, embedded in Tissue-Tek OCT Compound (Lab-Tek Products, Elkhart, IN, USA) and stored at $-70^{\circ} \mathrm{C}$ until further processing.

DESIGN OF MMP PRIMERS AND REVERSE TRANSCRIPTASE POLYMERASE CHAIN REACTION (RT-PCR)

For the design of the primers, sequences were searched in the NCBI Entrez search system (table 2). Sequence similarity search was done using the NCBI blastn program, and complementary search using Primers service in Williamstone Enterprises (http://www.williamstone. com/primers/index.html).

All samples were analysed for the mRNA expression of MMP-1 through MMP-20. Breast cancer, fibroblast, keratinocyte and bone marrow mRNA were used as positive controls. Finally, the full length cDNA for MMP-20 was obtained from odontoblastic cells $^{13}$ (table 2). Nine to 80 cryostat sections $(6 \mu \mathrm{m})$, depending on the sample size, were prepared for mRNA extraction using oligo(dT) $)_{25}$ covalently attached to magnetic polystyrene microbeads via 5' linker group (Dynabeads mRNA DIRECT kit, Dynal, Oslo, Norway). Extraction, performed according to the manufacturer's instructions, was controlled using RT-PCR for $\beta$-actin and spectrophotometric measurement. Sixty ng mRNA of each sample was used for first strand cDNA synthesis. This was performed with SuperScript Preamplification System, using oligo $(\mathrm{dT})_{12-18}$ for priming and RNase for removal of mRNA (GibcoBRL, Life Technologies, Paisley, Scotland, UK). cDNA synthesis without enzyme, without sample, or with control RNA were used as negative and positive controls, respectively. PCR amplification ${ }^{14}$ was performed using $0.2 \mu \mathrm{M}$ of target specific primers (table 2), $100 \mu \mathrm{M}$ of dATP, dCTP, dGTP and dTTP and $2 \mathrm{U}$ of the thermostable DNA polymerase (Finnzymes, Espoo, Finland) in $50 \mu$ of PCR buffer (10 mM TRIS-HCl, pH 8.8, $1.5 \mathrm{mM}$ $\mathrm{MgCl}_{2}, 50 \mathrm{mM} \mathrm{KCl}, 0.1 \%$ Triton X-100). The reaction was run in a thermal cycler (RoboCycler 40 Temperature Cycler, Stratagene, USA) for 40 cycles of one minute denaturation at $+95^{\circ} \mathrm{C}$, one minute annealing at the optimal temperature (table 2), one minute extension at $+72^{\circ} \mathrm{C}$ and finally 10 minutes extra extension for the last cycle. Amplifications without template, or without both template and primers, were performed as negative PCR controls. Extraction and RT-PCR were done at least twice for each sample. Amplified DNA was run on a $1 \%$ modified agarose gel (FMC

Table 2 Sequences of PCR primers, length of PCR product, optimal annealing temperature, sequence accession number from the NCBI Entrez search system and source of positive control

\begin{tabular}{|c|c|c|c|c|c|c|}
\hline & \multicolumn{2}{|l|}{ Primers $\left(5^{\prime}-3^{\prime}\right)$} & \multirow[b]{2}{*}{ Template bp } & \multirow[b]{2}{*}{ Annealing ${ }^{\circ} \mathrm{C}$} & \multirow{2}{*}{$\begin{array}{l}\text { Sequence } A C \\
\text { number }\end{array}$} & \multirow[b]{2}{*}{ Positive contro } \\
\hline & sense & antisense & & & & \\
\hline MMP-1 & CTGAAGGTGATGAAGCAGCC & AGTCCAAGAGAATGGCCGAG & 428 & 62 & $\mathrm{X} 05231$ & fibroblast \\
\hline MMP-2 & GCGACAAGAAGTATGGCTTC & TGCCAAGGTCAATGTCAGGA & 390 & 62 & J03210 & fibroblast \\
\hline MMP-3 & CTCACAGACCTGACTCGGTT & CACGCCTGAAGGAAGAGATG & 294 & 58 & J03209 & fibroblast \\
\hline MMP-7 & GTGGTCACCTACAGGATCGT & ACCATCCGTCCAGCGTTCAT & 282 & 62 & Z11887 & breast cancer \\
\hline MMP-8 & ATGGACCAACACCTCCGCAA & GTCAATTGCTTGGACGCTGC & 532 & 64 & J05556 & bone marrow \\
\hline MMP-9 & CGCAGACATCGTCATCCAGT & GGATTGGCCTTGGAAGATGA & 406 & 62 & J05070 & keratinocyte \\
\hline MMP-10 & GTCCTTCGATGCCATCAGCA & СTTGCTCCATGGACTGGCTA & 380 & 62 & X07820 & fibroblast \\
\hline MMP-11 & CAGGTGGCAGCCCATGAATT & GTACTGAGCACCTTGGAAGA & 456 & 58 & $\mathrm{X} 57766$ & breast cancer \\
\hline MMP-12 & CCACTGCTTCTGGAGCTCTT & GCGTAGTCAACATCCTCACG & 367 & 62 & L23808 & breast cancer \\
\hline MMP-13 & CTATGGTCCAGGAGATGAAG & AGAGTCTTGCCTGTATCCTC & 390 & 62 & $\mathrm{X} 75308$ & breast cancer \\
\hline MMP-14 & CAACACTGCCTACGAGAGGA & GTTCTACCTTCAGCTTCTGG & 380 & 60 & D26512 & breast cancer \\
\hline MMP-15 & GCATCCAGAACTACACGGAG & TACCGTAGAGCTGCTGGATG & 474 & 62 & Z48482 & breast cancer \\
\hline MMP-16 & TGTACCTGACCAGACAAGAG & AGTGTCCATGGCTCATCTGA & 384 & 58 & D50477 & breast cancer \\
\hline MMP-17 & GACCTGTTTGCAGTGGCTGT & ACGATCTTGTGGTCGCTGGT & 473 & 64 & X89576 & breast cancer \\
\hline MMP-19 & CAGGCTCTCTATGGCAAGAA & GAGCTGCATCCAGGTTAGGT & 397 & 62 & X92521 & breast cancer \\
\hline MMP-20 & GACCAGACCACAATGAACGT & GTCCACTTCTCAGGATTGTC & 374 & 62 & Y12779 & odontoblast \\
\hline$\beta$-actin & CACCTTCTACAATGAGCTGC & AGGCAGCTCGTAGCTCTTCT & 466 & 62 & X00351 & breast cancer \\
\hline
\end{tabular}


A $\begin{array}{llllllllllllllll}\mathrm{L} & \mathrm{N} & \mathrm{C} & \mathrm{R} & \mathrm{P} & 1 & 2 & 3 & 4 & 5 & 6 & 7 & 8 & 9 & 10 & \mathrm{~L}\end{array}$

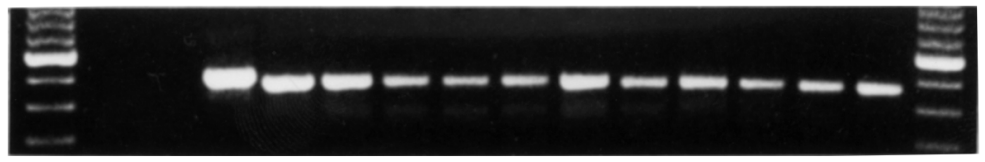

$\begin{array}{lllllllllllllllll}\mathrm{L} & \mathrm{M} & \mathrm{R} & \mathrm{N} & \mathrm{C} & \mathrm{P} & 1 & 2 & 3 & 4 & 5 & 6 & 7 & 8 & 9 & 10 & \mathrm{~L}\end{array}$

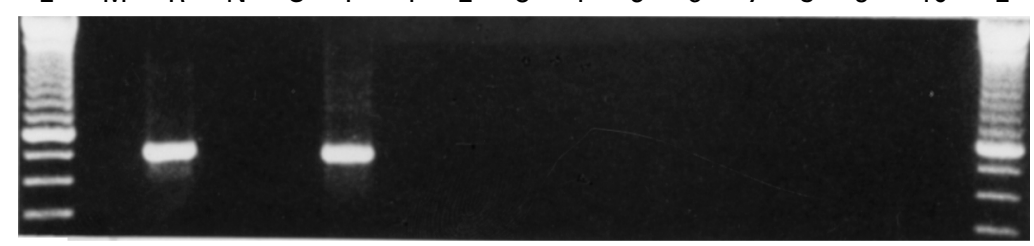

B

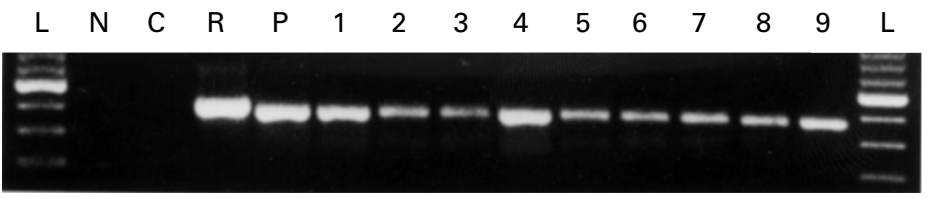

$\begin{array}{lllllllllllllllll}\mathrm{L} & \mathrm{M} & \mathrm{R} & \mathrm{N} & \mathrm{C} & \mathrm{P} & 1 & 2 & 3 & 4 & 5 & 6 & 7 & 8 & 9 & \mathrm{~L}\end{array}$

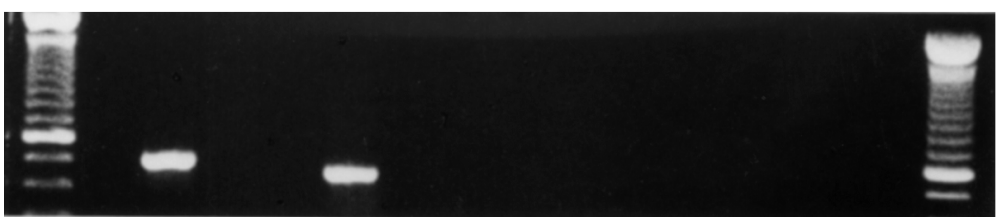

Figure 2 Reverse transcriptase polymerase chain reaction (RT-PCR) control analysis. $(A)$ Ten synovitis tissue samples from $R A$ patients (lanes 1-10). (B) Nine control non-arthritic knee injury samples (lanes 1-9). In both $(A)$ and (B), the upper panel shows $m R N A$ extraction control with $\beta$-actin PCR (466 bp) and the lower panel cDNA synthesis control with no RT enzyme (to exclude contamination with genomic DNA), but with regular PCR for $\beta$-actin. The expected size of the genomic $\beta$-actin would have been $907 \mathrm{bp}$. Samples are in same order. L indicates $100 \mathrm{bp} D N A$ ladder, starting from $300 \mathrm{bp} ; N$ for negative PCR control without sample and $C$ for negative PCR control without sample and primers were performed to exclude contamination of the reagents and found to be negative; $P$ for positive $P C R$ control of $\beta$-actin derived from breast cancer $c D N A ; M$ for negative $c D N A$ synthesis control without $R N A$ and $R$ for positive $c D N A$ synthesis control with control $R N A$ representing in vitro transcribed $R N A$ from the chloramphenicol acetyltransferase (CAT) gene provided with the kit (523 bp).

Bioproducts, ME, USA) and visualised with ethidium bromide for size verification. PCR fragments were extracted from the gel using silica gel membrane based QIAquick columns according to manufacturer's instructions (Qiagen Inc, Chatsworth, CA,USA) and quantified spectrophotometrically. Approximately $100 \mathrm{ng} /$ DNA fragment was sequenced using fluorescein labelled dye terminator kits (ABI, PE Applied Biosystems, Foster City, CA, USA) and analysed on Applied Biosystems automatic sequencer 373 A (PE Applied Biosystems, Foster City, CA, USA). ${ }^{15}$ One positive and one RA sample for each MMP were used for sequencing. The acquired sequence was verified with NCBI blastn program.

\section{Results}

MMP FAMILY

Altogether 16 different human MMP enzymes were identified (fig 1). Although numbered through 1 to 20, MMP-4, MMP-5 and MMP-6 have only been tentatively described, but to date have not been cloned and sequenced. MMP-18 is Xenopus collagenase- 4 , which does not have a human homologue. The most interesting finding was that the MMP-2/MMP-7 relation is closer than the MMP-2/MMP-9 relation when gelatin binding domain is left out.

MMP FAMILY MEMBERS AS IDENTIFIED IN TRAUMATIC AND RHEUMATOID SYNOVIAL MEMBRANE

Messenger RNA extraction, as demonstrated using $\beta$-actin PCR, was successful for all RA $(\mathrm{n}=10$, fig 2, panel $\mathrm{A}$, upper part) and all trauma samples $(n=9$, fig 2 , panel $B$, upper part). cDNA synthesis was controlled by experiments, in which no RT enzyme was used and showed that the PCR products were derived from RNA (fig 2, panels A and B, lower parts). The expected size of the genomic $\beta$-actin would have been $907 \mathrm{bp}$. Negative controls for cDNA synthesis without RNA were negative and positive controls for cDNA synthesis with control RNA (523 bp), representing in vitro transcribed RNA from the chloramphenicol acetyltransferase (CAT) gene provided with the kit, were positive. Negative PCR controls, without sample and primers, or without sample, performed to exclude contamination of the reagents, were negative. Positive PCR control of $\beta$-actin derived from breast cancer cDNA was positive. All samples were analysed for the MMP mRNA (fig 3, table 3).

In addition to the size and sequence verified specific MMP bands, MMP-15 gels consistently showed an extra band below the $474 \mathrm{bp}$ MMP-15 amplicon and MMP-19 an extra band above the $397 \mathrm{bp}$ MMP-19 amplicon. The sequence of the last mentioned extra band was at the 3'-end the same as that of the MMP-19, but the extra band contained an approximately $100 \mathrm{bp}$ insert in its middle. It is unclear at present whether these extra bands represent results of alternative splicing, new MMPs or something else. However, they do not represent genomic DNA, because the controls for this were negative.

Briefly, the results can be summarised as follows, when expressed as the number of positive samples / total number of samples:

Group $A$ (high \% of positive samples in both conditions): MMP-2 (gelatinase A; 9 of 9 in trauma, 10 of 10 in RA), MMP-3 (stromelysin-1; 8 of 9 in trauma, 10 of 10 in RA), MMP-11 (stromelysin-3; 9 of 9 in trauma, 10 of 10 in RA) and MMP-19 (8 of 9 in trauma, 10 of 10 in RA). These MMPs seemed to be constitutively expressed.

Group $B$ MMP-1 (fibroblast type collagenase), MMP-9 (gelatinase B) and MMP-14 (MT1-MMP) were always, without exception, expressed in RA samples, but were found to be positive in the control samples to a lower extent as follows: MMP-1 in 6 of 9, MMP-9 in 5 of 9 and MMP-14 in 7 of 9 cases.

Group $C$ (exclusive expression in RA samples): MMP-13 (collagenase-3; 9 of 10 in RA versus 0 of 9 in trauma) and MMP-15 (MT2MMP; 8 of 10 in RA versus 0 of 9 in trauma) 
A $\begin{array}{lllllllllllllll}\mathrm{L} & \mathrm{N} & \mathrm{C} & \mathrm{P} & 1 & 2 & 3 & 4 & 5 & 6 & 7 & 8 & 9 & 10 & \mathrm{~L}\end{array}$ MMP-1

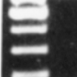

MMP-2

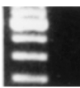

MMP-3

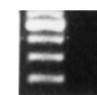

MMP-7

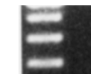

MMP-8

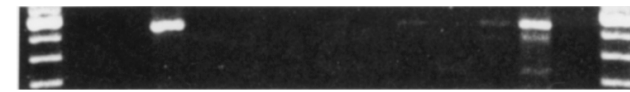

MMP-9

MMP-10
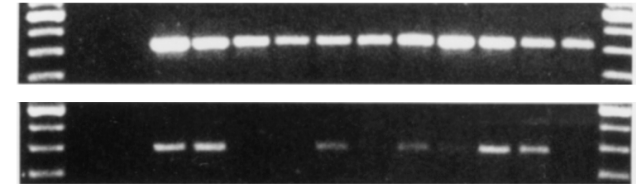

MMP-11

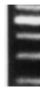

MMP-12
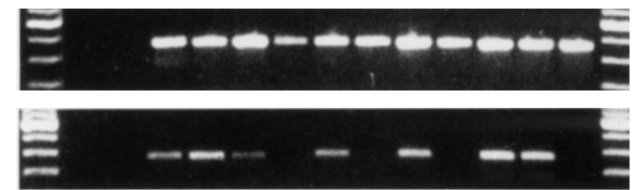

MMP-13

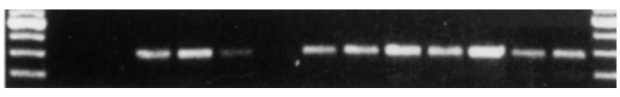

MMP-14

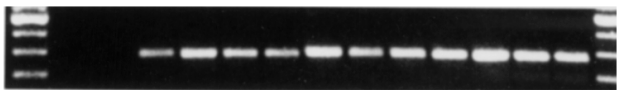

MMP-15

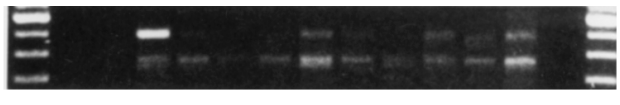

MMP-16

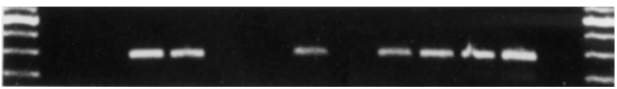

MMP-17

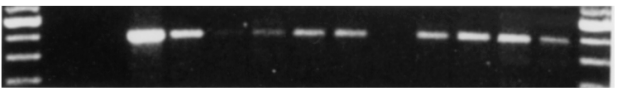

MMP-19

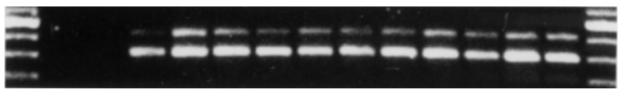

MMP-20

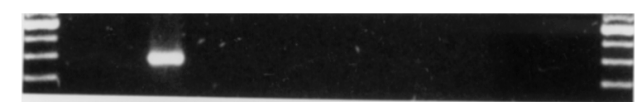

B

$\begin{array}{llllllllllllll}\mathrm{L} & \mathrm{N} & \mathrm{C} & \mathrm{P} & 1 & 2 & 3 & 4 & 5 & 6 & 7 & 8 & 9 & \mathrm{~L}\end{array}$
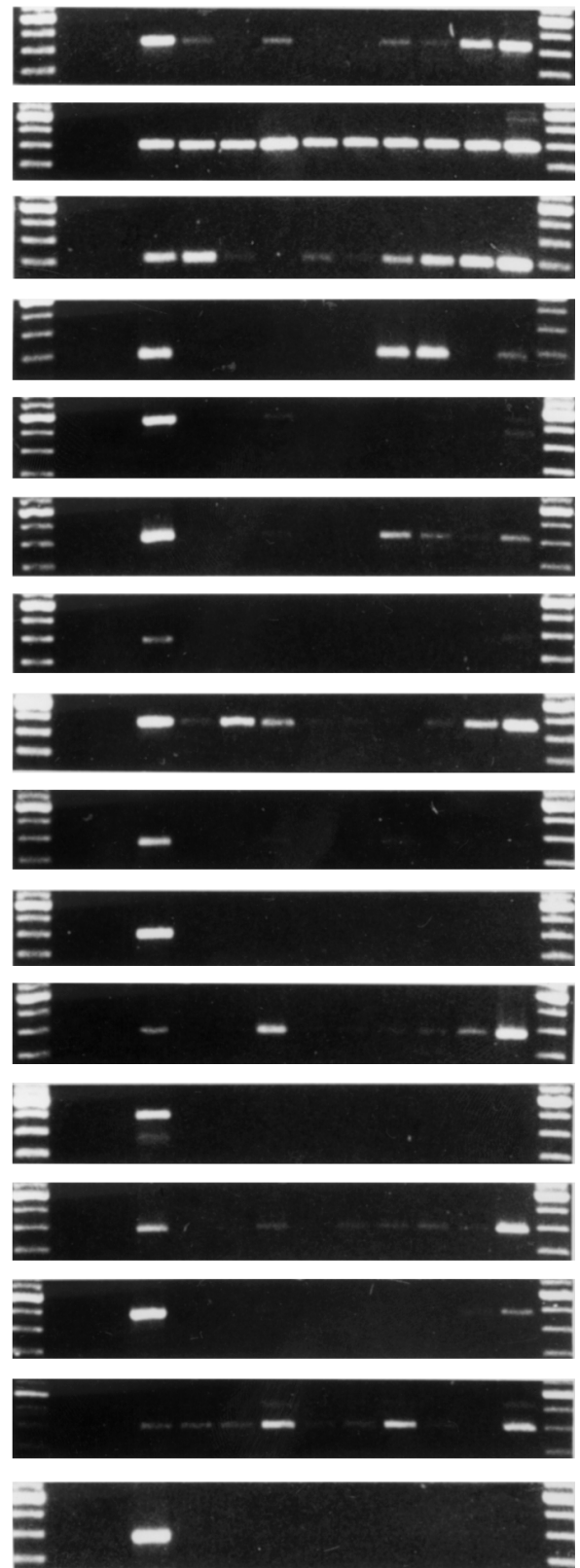

Figure 3 Reverse transcriptase polymerase chain reaction (RT-PCR) analysis of MMP-1 through MMP-20. (A) Ten synovitis tissue samples from RA patients (lanes 1-10). (B) Nine control non-arthritic knee injury samples (lanes 1-9). Samples are in the same order as in figure 2. It should be noted that the weakest bands are not visible in the photographic reproductions. Therefore, all data given in the results section and in table 3 are based on observations made from the original gels and photographs. L indicates $100 \mathrm{bp} D N A$ ladder, starting from $300 \mathrm{bp} ; N$ for negative PCR control without sample; C for negative PCR control without sample and primers; and P for positive PCR control.

were only expressed in RA, but were not found in any of the control samples.

Group D (complete lack or low \% of positive samples): MMP-20 (enamelysin; 0 of 0 in RA, 0 of 0 in trauma) was not found at all, although the probes worked very well for the positive MMP-20 control. ${ }^{13}$ MMP-8 (collagenase-2) was rarely found in traumatic (3 of 9) and rheumatoid (4 of 10) samples.

Group $E$ All the other MMPs had an intermediate pattern of expression (table 3), usually with a slight predominance in RA compared with trauma (MMP-7: 70\% versus 33\%; MMP-10: $60 \%$ versus 11\%; MMP-12: $60 \%$ versus $33 \%$; MMP-16: $60 \%$ versus $77 \%$; MMP-17: 90\% versus 33\%).
ASSOCIATION OF THE MMP PROFILE WITH THE SYNOVIAL MEMBRANE HISTOLOGY AND

MEDICATION OF THE PATIENTS

Samples were separately and blindly scored to those containing no $(-)$, mild $(+)$, moderate $(++)$ or strong $(+++)$ mononuclear cell infiltrates. The evaluation of mononuclear cell infiltration was as follows: $-=$ no histological signs of mononuclear inflammatory cell infiltrates; $+=$ only occasional and small inflammatory cell infiltrates; $++=$ numerous perivascular cell infiltrates; $+++=$ many tight perivascular and diffuse inflammatory cell infiltrates. ${ }^{16}$

Only one of the controls, a 42 year old man, who had had a subluxation of patella, had mild 
Table 3 Matrix metalloproteinase (MMP) family members as found in the traumatic and rheumatoid synovial membrane. MMPs are identified according to the MMP nomenclature and their synonyms. Results of the RT-PCR are given as the number of positive samples per all samples tested

\begin{tabular}{llll}
\hline & & \multicolumn{2}{l}{ Positive samples/all } \\
\cline { 3 - 4 } MMP type & Synonyms & $R A$ & Control \\
\hline MMP-1 & Fibroblast type collagenase, collagenase-1 & $10 / 10$ & $6 / 9$ \\
MMP-2 & Gelatinase A, 72 kDa type IV collagenase & $10 / 10$ & $9 / 9$ \\
MMP-3 & Stromelysin-1, transin-1, proteoglycanase & $10 / 10$ & $8 / 9$ \\
MMP-7 & Matrilysin, uterine metalloproteinase, PUMP-1 & $7 / 10$ & $3 / 9$ \\
MMP-8 & Neutrophil type collagenase, collagenase-2 & $4 / 10$ & $3 / 9$ \\
MMP-9 & Gelatinase B, 92 kDa type IV collagenase & $10 / 10$ & $5 / 9$ \\
MMP-10 & Stromelysin-2, transin-2 & $6 / 10$ & $1 / 9$ \\
MMP-11 & Stromelysin-3 & $10 / 10$ & $9 / 9$ \\
MMP-12 & Macrophage metalloelastase & $6 / 10$ & $3 / 9$ \\
MMP-13 & Collagenase-3 & $9 / 10$ & $0 / 9$ \\
MMP-14 & MT1-MMP & $10 / 10$ & $7 / 9$ \\
MMP-15 & MT2-MMP & $8 / 10$ & $0 / 9$ \\
MMP-16 & MT3-MMP & $6 / 10$ & $7 / 9$ \\
MMP-17 & MT4-MMP & $9 / 10$ & $3 / 9$ \\
MMP-19 & & $10 / 10$ & $8 / 9$ \\
MMP-20 & Enamelysin & $0 / 0$ & $0 / 0$ \\
\hline
\end{tabular}

mononuclear cell infiltrates in his knee synovial membrane. His MMP profile was different from the other controls and very reminescent of the RA MMP profile in that he expressed all except three MMPs. Interestingly, those that were not expressed were MMP-13, MMP-15 (MT2MMP) and MMP-20. Three of the RA samples contained only mild mononuclear cells infiltrates and those patients had the lowest levels of MMPs: one did not express MMP-7, MMP-8, MMP-10, MMP-12, MMP-13, MMP-16 or MMP-20, one did not express MMP-7, MMP-8, MMP-10, MMP-12, MMP-16 or MMP-20 and one did not express MMP-8, MMP-10, MMP-12, MMP-15, MMP-16 or MMP-20. In contrast with the association with the degree of synovial inflammation, there were no clear cut associations between the MMP profile and medication, although disease modifying, anti-rheumatic drugs can affect MMP gene expression. ${ }^{17-19}$ Synovial samples were taken from different joints and from different regions of the synovial tissue. These differences could have a dramatic effect on the expression of MMPs. However, the same pattern of MMP expression emerged from all joints affected by $\mathrm{RA}$. It seems, that the rheumatoid disease process rather than the site of the sample is decisive as to what type of MMP profile will be expressed at the mRNA level.

\section{Discussion}

Some of the MMPs seem to be expressed in all or almost all traumatic synovial membrane samples. Traumatic synovial membrane is naturally not normal synovial membrane. However, this may imply a constitutive expression pattern and a role in normal tissue remodelling processes. These MMPs were MMP-2 (gelatinase A or 72 $\mathrm{kDa}$ type IV collagenase), MMP-3 (stromelysin1), MMP-11 (stromelysin-3) and MMP-19. Their constitutive expression does not mean that they could not play a part in pathological tissue destruction, for example, because of their increased synthesis in diseases and/or insufficient regulation of their degradative potential. For example, MMP-2 has recently been found to be part of a cell membrane associated ternary MT1-MMP/TIMP-2/MMP-2 complex, which may contribute to proMMP-2 activation and focused, pericellular targeting of its action. ${ }^{20}$ In addition, MMP-2 is, not only gelatinase, but also a collagenase active against interstitial type I and II collagens. ${ }^{21} 22$

Some MMP mRNAs were found in all RA synovial tissue samples, namely MMP-1 (fibroblast type collagenase or collagenase-1), MMP-9 (gelatinase $\mathrm{B}, 92 \mathrm{kDa}$ type IV collagenase) and MMP-14 (MT1-MMP). Indeed, MMP-1 and MMP-9 are known to be induced by cytokines like interleukin 1 (IL1), tumour necrosis factor $\alpha(\mathrm{TNF} \alpha)$, interferon $\gamma$, transforming growth factor $\alpha$ and epidermal growth factor. In this respect the two "gelatinases", MMP-2 and MMP-9, differ in that MMP-2 is constitutively expressed and relatively unresponsive to most growth factors and cytokines, whereas MMP-9 is quite responsive. Less is known on the regulation of MT1-MMP gene transcription. Okada and coworkers have shown IL $1 \alpha$ and/or TNF $\alpha$ driven MT1-MMP induction in chondrocytes. ${ }^{23}$ In RA synovial membrane MT1-MMP seems to follow in its expression the same pattern as MMP-1 and MMP-9.

MMP-20 (enamelysin) was not expressed in any of the trauma or RA samples. MMP-8 (neutrophil type collagenase, collagenase-2) was only found in 4 of 10 RA and 3 of 9 trauma samples. The formerly used term "neutrophiltype collagenase" seems to be a misnomer. It was originally considered to be neutrophil specific. MMP-8 is synthesised during the myelocyte stage of development and stored in the specific or secondary granules to be released upon neutrophil activation. More recently, it has been shown that chondrocytes ${ }^{24}$ and TNF $\alpha$ stimulated rheumatoid synovial fibroblasts ${ }^{25}$ synthesise a less glycosylated, mesenchymal form of MMP-8, which is occasionally also found in rheumatoid synovial membrane.

MMP-13 or collagenase-3 was not found in any of the trauma samples, but was found in almost all (9 of 10) rheumatoid samples. This is in accordance with earlier observations, which demonstrate MMP-13 enzyme protein in immunohistochemical staining in RA to a much higher extent than it was found in osteoarthritis and that the expression of MMP-13 seems to correlate with the degree of inflammation. ${ }^{26}{ }^{27}$ IL1 $\beta$ and $\mathrm{TNF} \alpha$ induce fibroblasts to synthesise and secrete MMP-13. ${ }^{28}$ These cytokines may affect the tetradecanoylphorbol acetate (TPA) responsive TRE element of the collagenase- 3 gene, because also TPA leads to stimulation of fibroblast mediated collagenase- 3 synthesis. ${ }^{29} 30$ It seems therefore, that inflammatory cytokines with a central role in arthritis-that is, IL1 $\beta$ and $\mathrm{TNF} \alpha$, induce MMP-13 gene transcription and expression in the RA synovial membrane.

Another enzyme, which was not found in any of the trauma samples, but was found in 8 of 10 RA samples, was MMP-15 or MT2-MMP. MT2-MMP is a member of the membranetype MMP subfamily. MT2-MMP can degrade fibronectin, tenascin, nidogen, aggrecan, perlecan and laminin, process pro-TNF $\alpha$ to a 
mature form and activate proMMP-2. ${ }^{31} 32$ Although gene regulation of MT2-MMP has not been studied yet, the present results suggest that, like MMP-13, MT2-MMP is induced in inflammation. Because of its direct degradative potential and its activator role for MMPs and $\mathrm{TNF} \alpha,{ }^{31}{ }^{32}$ it may play an important part in the MMP mediated, proteolytic processes. However, it should be noted that mRNA expression is not equivalent with a subsequent translation of the mRNA to the corresponding enzyme protein and does not necessarily mean that the latent proenzyme zymogen synthesised is secreted and activated. Furthermore, regulation of the activated MMP enzyme species by their endogenous inhibitors, such as TIMPs or $\alpha_{2}$-macroglobulin, adds more to the complexity of the situation. Therefore, much work remains to be done until it is possible to understand the MMP mediated local tissue destructive processes in any detail. This study gives some solid foundation for future work in demonstrating, which of the MMPs are locally transcribed and which are not.

In conclusion, there are distinct differences in the expression of MMPs in traumatic and rheumatoid synovial membranes. Some MMPs are not expressed at all (MMP-20) or are expressed only rarely (MMP-8) in any synovial tissue samples whether inflamed or not, some are "constitutively" expressed in both trauma and RA (MMP-2, MMP-3, MMP-11 and MMP-19), whereas some are only (MMP-13, MMP-15) or predominantly (MMP-1, MMP-9, MMP-14) seen in RA. The rest of the MMPs have an intermediate pattern of expression (MMP-7, MMP-10, MMP-12, MMP-16, MMP-17) usually with a slight predominance in RA. Despite clear differences in the MMP profiles of RA and "non-inflammatory" controls, the synovial MMP network responsible for remodelling and inflammatory tissue destruction may be more complex than has been anticipated. Nevertheless, the excessive expression of some MMPs in the RA synovial membrane may facilitate the development of selective diagnostic and therapeutic measures.

1 Springman EB, Angleton EL, Birkedal-Hansen H, van Wart HE. Multiple modes of activation of latent human fibroblast collagenase: evidence for the role of Cys 73 active fibroblast collagenase: evidence for the role of Cys 73 active site zinc complex in latency and a "cysteine switch" mechanism for

2 Evanson JM, Jeffrey JJ, Krane SM. Human collagenase: identification and characterization of an enzyme from rheumatoid synovium in culture. Science 1967;158:499502 .

3 Chatham WW, Heck LW, Blackburn WD Jr. Lysis of fibrillar collagen by neutrophils in synovial fluid. A role for surface-bound immunoglobulins. Arthritis Rheum 1990; 33:1333-9.

4 Konttinen YT, Lindy O, Kemppinen P, Saari H, Suomalainen $\mathrm{K}$, Vauhkonen $\mathrm{M}$, et al. Collagenase reserves in polymorphonuclear neutrophil leukocytes from synovial fluid and peripheral blood of patients with rheumatoid arthritis. Matrix 1991;11:296-301.

5 Case JP, Lafyatis R, Remmers EF, Kumkumian GK, Wilde Case JP, Lafyatis R, Remmers EF, Kumkumian GK, Wilde RL. Transin/stromelysin expression in rheumatoid synovium. A transformation- associated metalloproteinase secreted by phenotypically

6 Okada Y, Takeuchi N, Tomita K, Nakanishi I, Nagase H. Immunolocalization of matrix metalloproteinase 3 (stromelysin) in rheumatoid synovioblasts (B cells): correlation with rheumatoid arthritis. Ann Rheum Dis 1989;48: 645-53.

7 Stolow MA, Bauzon DD, Li J, Sedgwick T, Liang VC, Sang QA, Shi YB. Identification and characterization of a novel collagenase in Xenopus laevis: possible roles during frog development Mol Biol Cell 1996; 7:1471-83.

8 Cossins J, Dudgeon TJ, Catlin G, Gearing AJ, Clements JM Identification of MMP-18, a putative novel human matrix metalloproteinase. Biochem Biophys Res Commun 1996; 228:494-8.

9 Pendas AM, Balbin M, Llano E, Jimenez MG, López-Otín C. Structural analysis and promoter characterization of the human collagenase-3 gene (MMP13). Genomics 1997;40: 222-33.

10 Feng DF, Doolittle RF. Progressive sequence alignment as prerequiste to correct phylogenetic trees. J Mol Evol 1987; 25:351-60

11 Higgins DG, Sharp PM. Fast and sensitive multiple sequence alignment on a microcomputer. Comp Appl Biosci 1989;5:151-3.

12 Arnett FC, Edworthy SM, Bloch DA, McShane DJ, Fries JF, Cooper NS, et al. The American Rheumatism Association 1987 revised criteria for the classification of rheumatoid arthritis. Arthritis Rheum 1988;31:315-24.

13 Llano E, Pendas AM, Knäuper V, Sorsa T, Salo T, Salido E, et al. Identification and structural and functional characterization of human enamelysin (MMP-20). Biochemistry terization of human

14 Innis MA, Gelfand DH, Sninsky JJ, White TJ. PCR protocols: a guide to methods and applications. San Diego, CA: Academic Press, 1990.

15 Kristo P, Saarelainen R, Fagerström R, Aho S, Korhola M Protein purification and cloning and characterization of the cDNA and gene for xylose isomerase of barley. Eur J Biochem 1996;237:240-6.

16 Konttinen YT, Bergroth V, Nordström D, Koota K, Skrifvars B, Hagman G, et al. Cellular immunohistopathology of acute, subacute and chronic synovitis in rheumatoid ogy of acute, subacute and chronic synovitis
arthritis. Ann Rheum Dis 1985;44:549-55.

17 Makino Y, Tanaka H, Makino I. Paradoxical derepression of collagenase gene expression by the antirheumatic gold compound aurothiomalate. Mol Pharmacol 1994;46: 1084-9.

18 Firestein GS, Paine MM, Boyle DL. Mechanisms of methotrexate action in rheumatoid arthritis. Selective decrease in synovial collagenase gene expression. Arthritis Rheum 1994;37:193-200.

19 Lohi I, Kähäri VM, Keski-Oja J. Cyclosporin A enhances cytokine and phorbol ester-induced fibroblast collagenase expression. J Invest Dermatol 1994;102:938-44.

20 Strongin AY, Collier I, Bannikov G, Marmer BL, Grant GA, Goldberg GI. Mechanism of cell surface activation of $72-\mathrm{kDa}$ type IV collagenase. Isolation of the activated form of the membrane metalloprotease. J Biol Chem 1995;270: of the mem $5331-8$.

21 Aimes RT, Quigley JP. Matrix metalloproteinase-2 is an interstitial collagenase. J Biol Chem 1995;270:5872-6.

22 Konttinen YT, Ceponis A, Takagi M, Ainola M, Sorsa T, Sutinen $M$, et al. New collagenolytic enzymes/cascade identified at the pannus-hard tissue junction in rheumatoid arthritis: destruction from above. Matrix Biol 1998;17: 585-601.

23 Imai K, Ohta S, Matsumoto T, Fujimoto N, Sato H, Seiki $\mathrm{M}$, et al. Expression of membrane-type 1 matrix metalloproteinase and activation of progelatinase $\mathrm{A}$ in human proteinase and activation of progelatinase A in hum

24 Cole AA, Chubinskaya S, Schumacher B, Huch K, Szabo G, Yao J, et al. Chondrocyte matrix metalloproteinase- 8 . Human articular chondrocytes express neutrophil collaHuman articular chondrocytes express
genase. J Biol Chem 1996;271:11023-6.

25 Hanemaaijer R, Sorsa T, Konttinen YT, Ding Y, Sutinen M, Visser $\mathrm{H}$, et al. Matrix metalloproteinase-8 is expressed in rheumatoid synovial fibroblasts and endothelial cells Regulation by tumor necrosis factor- $\alpha$ and doxycycline. Biol Chem 1997;272:31504-9.

26 Lindy O, Konttinen YT, Sorsa T, Ding Y, Santavirta S, Ceponis A, et al. Matrix metalloproteinase 13 (collagenase-3) in human rheumatoid synovium. Arthritis Rheum 1997;40:1391-9.

27 Imai S, Konttinen YT, Jumppanen M, Lindy O, Čeponis A, Kemppinen P, et al. High levels of expression of collagenase-3 (MMP-13) in pathological conditions associated to a foreign body reaction. J Bone Joint Surg 1998;80B: $701-10$

28 Uria JA, Stahle-Backdahl M, Seiki M, Fueyo A, López-Otín C. Regulation of collagenase-3 expression in human breast carcinomas is mediated by stromal-epithelial cell interactions. Cancer Res 1997;57:4882-8

29 Pendas AM, Knäuper V, Puente XS, Llano E, Mattei MG Apte $S$, et al. Identification and characterization of a novel human matrix metalloproteinase with unique structural characteristics, chromosomal location, and tissue distribution. J Biol Chem 1997a;272:4281-6.

30 Tardif G, Pelletier JP, Dupuis M,Hambor JE, MartelPelletier J. Cloning, sequencing and characterization of the 5'-flanking region of the human collagenase-3 gene. 5 -flanking region of the
Biochem J 1997;323:13-16.

31 d'Ortho MP, Will H, Atkinson S, Butler G, Messent A, Gavrilovic J, et al. Membrane-type matrix metalloproteinases 1 and 2 exhibit broad-spectrum proteolytic capacities comparable to many matrix metalloproteinases. Eur J Biochem 1997;250:751-77.

32 Kolkenbrock H, Hecker-Kia A, Orgel D, Ulbrich N, Will H. Activation of progelatinase $\mathrm{A}$ and progelatinase A/TIMP-2 complex by membrane type 2 - matrix metalloproteinases. Biol Chem 1997;378:71-6. 